\title{
ON THE RETARDED LIÉNARD EQUATION
}

\author{
BO ZHANG
}

(Communicated by Kenneth R. Meyer)

\begin{abstract}
We consider the equation $x^{\prime \prime}+f(x) x^{\prime}+g(x(t-h))=0$ in which $f$, $g$ are continuous with $f(x)>0$ for $x \in R, h$ is a nonnegative constant, and $x g(x)>0$ if $|x| \geq k$ for some $k \geq 0$. Necessary and sufficient conditions are given for boundedness of all solutions and their derivatives. When $k=0$ we give necessary and sufficient conditions for all solutions and their derivatives to converge to zero.
\end{abstract}

We consider the retarded Liénard Equation

$$
x^{\prime \prime}+f(x) x^{\prime}+g(x(t-h))=0
$$

in which $h$ is a nonnegative constant and $g, f$ are continuous with $f(x)>0$ for all $x \in R$; it is supposed that exist constants $k \geq 0$ and $N>1$ such that

$$
x g(x)>0, \quad x\left(\int_{0}^{x} f(\xi) d \xi-N h g(x)\right)>0 \quad \text { if }|x| \geq k .
$$

Equation (1), with or without delay, has attracted considerable attention for more than fifty years, particularly in the theory of asymptotic behavior of solutions (cf. [1-16]). In 1965 Burton [3] obtained the following result:

Theorem B. Suppose that $f$ and $g$ are continuous with $f(x)>0, x g(x)>0$ if $x \neq 0$. Then the zero solution of (1) with $h=0$ is globally asymptotically stable if and only if

$$
\int_{0}^{ \pm \infty}[f(x)+|g(x)|] d x= \pm \infty .
$$

Much effort has gone into the modification of signum conditions on $f$ and $g$ when boundedness of solutions is considered (cf. $[4,6,12,13,14,15]$ ).

For the retarded equation (1), it is often assumed that

$$
\left|g^{\prime}(x)\right| \leq L \text { for some constant } L>0
$$

and

$$
x g(x)>0 \quad(x \neq 0), \quad f(x)>h L
$$

Received by the editors December 17, 1990.

1991 Mathematics Subject Classification. Primary 34K25, 34D20.

Key words and phrases. Necessary and sufficient conditions, boundedness and global asymptotic stability.

Presented at the Nineteenth Midwest Conference on Differential Equations, October 1990, University of Missouri at Rolla, Missouri. 
in order to obtain the results on boundedness and stability (cf. $[2,9,16])$. It is clear that $(2)$ is a generalization of $\left(4^{*}\right)$.

We consider the following questions: Is condition (3) still necessary and sufficient for boundedness and stability of solutions of (1)? For the case $h>$ 0 , is Theorem B still valid when condition (4) is not satisfied? We have an affirmative answer to these questions.

A system equivalent to (1) is

$$
\left\{\begin{array}{l}
x^{\prime}=y \\
y^{\prime}=-g(x(t-h))-f(x) y .
\end{array}\right.
$$

Notice that condition (2) guarantees the global existence of solutions of system (5) for increasing $t$. Let $R^{+}, R$ denote the intervals $[0,+\infty)$ and $(-\infty,+\infty)$ respectively. If $x$ or $y$ is written without its argument then the argument is $t$. We also denote by $V^{\prime}(t)$ the upper right-hand derivative of $V(t)$ with respect to $t$ if no confusion occurs.

Theorem 1. If (2) is satisfied, then all solutions of (5) are bounded if and only if (3) holds.

Proof. Define $F(x)=\int_{0}^{x} f(\xi) d \xi, \quad G(x)=\int_{0}^{x} g(\xi) d \xi$, and let $x(t)=$ $x\left(t, \phi, y_{0}\right), y(t)=y\left(t, \phi, y_{0}\right)$ be a solution of (5) with $\phi \in C([-h, 0], R)$, $x(\xi)=\phi(\xi)$ for $\xi \in[-h, 0]$, and $y(0)=y_{0}$; then $(x(t), y(t))$ exists on $R^{+}$. We now define

$$
\begin{aligned}
V_{0}(t)= & {\left[y(t)+F(x(t))-\int_{-h}^{0} g(x(t+s)) d s\right]^{2}+2 G(x(t)) } \\
& +N \int_{-h}^{0} \int_{t+s}^{t}|g(x(\tau))|^{2} d \tau d s+P
\end{aligned}
$$

where $P=2 \sup \{|G(\xi)|:|\xi| \leq k\}$; then

$$
\begin{aligned}
V_{0}^{\prime}(t) \leq & -2(F(x(t))-N h g(x(t))) g(x(t))-(N-1) h|g(x(t))|^{2} \\
& -(N-1) \int_{-h}^{0}|g(x(t+s))|^{2} d s .
\end{aligned}
$$

Next define

$$
V_{1}(t)=V_{0}(t)+\frac{1}{2}(N-1) h \int_{-h}^{0}|g(x(t+s))|^{2} d s
$$

then

$$
\begin{aligned}
V_{1}^{\prime} \leq & -2(F(x(t))-N h g(x(t))) g(x(t))-\frac{1}{2}(N-1) h|g(x(t))|^{2} \\
& -(N-1) \int_{-h}^{0}|g(x(t+s))|^{2} d s \\
= & -\frac{3}{2}(F(x)-N h g(x)) g(x)-\frac{1}{2 N}(N-1) F(x) g(x) \\
& -\frac{1}{2 N}(F(x)-N h g(x)) g(x)-(N-1) \int_{-h}^{0}|g(x(t+s))|^{2} d s \\
\leq & -(N-1) F(x) g(x) / 2 N \text { if }|x| \geq k .
\end{aligned}
$$


Now define

$$
V_{2}(t)=|y(t)|+\int_{-h}^{0}|g(x(t+s))| d s .
$$

We then have

$$
V_{2}^{\prime}(t) \leq-f(x(t))|y(t)|+|g(x(t))| .
$$

We will use the following constants

$$
\begin{gathered}
f_{0}=\min \{f(x):|x| \leq k\}, \quad g^{*}=\max \{|g(x)|:|x| \leq k\}, \\
F_{0}=\min \{F(k),|F(-k)|\}, \quad F^{*}=\max \{F(k),|F(-k)|\}, \\
\gamma=F_{0}(N-1) / 4 N .
\end{gathered}
$$

Finally we define

$$
V(t)=V_{1}(t)+\gamma V_{2}(t)
$$

then

$$
\begin{aligned}
V^{\prime}(t) \leq & -2(F(x(t))-N h g(x(t))) g(x(t))-\frac{1}{2}(N-1) h|g(x(t))|^{2} \\
& -(N-1) \int_{-h}^{0}|g(x(t+s))|^{2} d s-\gamma f(x)|y(t)|+\gamma|g(x)| .
\end{aligned}
$$

Choose $Q>k$ such that $3 g^{*} F^{*}+2 N h\left[g^{*}\right]^{2}-\gamma f_{0}(Q-k)<-1$ and consider $|x(t)|+|y(t)| \geq Q$ :

If $|x(t)| \geq k$ then

$$
\begin{aligned}
V^{\prime}(t) & \leq-\frac{1}{2 N}(N-1) F(x) g(x)-\gamma f(x)|y(t)|+\gamma|g(x)| \\
& \leq-\gamma f(x)|y(t)|-(N-1) F(x) g(x) / 4 N .
\end{aligned}
$$

If $|x(t)|<k$ then $|y(t)| \geq Q-k$ and

$$
\begin{aligned}
V^{\prime}(t) & \leq 2|F(x)||g(x)|+2 N h|g(x)|^{2}+\gamma|g(x)|-\gamma f(x)|y(t)| \\
& \leq 2 F^{*} g^{*}+2 N h\left[g^{*}\right]^{2}+\gamma\left|g^{*}\right|-\gamma f_{0}(Q-k)<-1 .
\end{aligned}
$$

Thus

$$
V^{\prime}(t)<0 \quad \text { if }|x(t)|+|y(t)| \geq Q
$$

Notice also that

$$
\begin{aligned}
V^{\prime}(t) \leq & -2|F(x)-N h g(x)||g(x)|-(N-1) h|g(x)|^{2} / 4 \\
& -(N-1) \int_{-h}^{0}|g(x(t+s))|^{2} d s+M,
\end{aligned}
$$

where $M=4 \max \{|F(\xi)-N h g(\xi)||g(\xi)|:|\xi| \leq k\}+(N-1) F_{0}^{2} / 16 h N^{2}$. If there exists $t_{1}>0$ such that $V(0)<V\left(t_{1}\right)$ and

$$
V\left(t_{1}\right)=\max _{0 \leq s \leq t_{1}} V(s),
$$

then

$$
\left|x\left(t_{1}\right)\right|+\left|y\left(t_{1}\right)\right|<Q
$$


and

$$
\frac{1}{4}(N-1) h\left|g\left(x\left(t_{1}\right)\right)\right|^{2}+(N-1) \int_{t_{1}-h}^{t_{1}}|g(x(s))|^{2} d s \leq M .
$$

By (10) it follows that

$$
\begin{aligned}
V\left(t_{1}\right)= & {\left[y\left(t_{1}\right)+F\left(x\left(t_{1}\right)\right)-\int_{t_{1}-h}^{t_{1}} g(x(s)) d s\right]^{2}+2 G\left(x\left(t_{1}\right)\right) } \\
& +N \int_{-h}^{0} \int_{t_{1}+s}^{t_{1}}|g(x(\nu))|^{2} d \nu d s+\frac{1}{2}(N-1) h \int_{t_{1}-h}^{t_{1}}|g(x(s))|^{2} d s+P \\
& +\gamma\left|y\left(t_{1}\right)\right|+\gamma \int_{-h}^{0}|g(x(t+s))| d s \\
\leq & 2\left[y\left(t_{1}\right)+F\left(x\left(t_{1}\right)\right)\right]^{2}+2\left(\int_{-h}^{0} g\left(x\left(t_{1}+s\right)\right) d s\right)^{2}+2 G\left(x\left(t_{1}\right)\right) \\
& +N h \int_{t_{1}-h}^{t_{1}}|g(x(s))|^{2} d s+\frac{1}{2}(N-1) h \int_{t_{1}-h}^{t_{1}}|g(x(s))|^{2} d s+P \\
& +\gamma\left|y\left(t_{1}\right)\right|+\gamma \int_{-h}^{0}\left|g\left(x\left(t_{1}+s\right)\right)\right|^{2} d s+\gamma h \\
\leq & 4\left|y\left(t_{1}\right)\right|^{2}+4\left|F\left(x\left(t_{1}\right)\right)\right|^{2}+2 h \int_{-h}^{0}\left|g\left(x\left(t_{1}+s\right)\right)\right|^{2} d s+P \\
& +2\left|G\left(x\left(t_{1}\right)\right)\right|+\frac{2 h N M}{N-1}+\gamma Q+\frac{\gamma M}{N-1}+\gamma h \\
\leq & 4\left|F\left(x\left(t_{1}\right)\right)\right|^{2}+2\left|G\left(x\left(t_{1}\right)\right)\right|+P+4 Q^{2}+\frac{2 h M}{N-1} \\
& +\frac{2 N h M}{N-1}+\gamma Q+\frac{\gamma M}{N-1}+\gamma h .
\end{aligned}
$$

Let $\bar{K}=\max \left\{|F(\xi)|^{2}+|G(\xi)|:|\xi| \leq Q\right\}$; then

$$
V\left(t_{1}\right) \leq 6 \bar{K}+4 Q^{2}+\frac{(2 h+2 N h+\gamma) M}{N-1}+P+\gamma(Q+h) \stackrel{\text { def }}{=} B .
$$

Hence,

$$
V(t) \leq V(0)+B \text { for all } t \in R^{+} .
$$

Consequently, we have $|y(t)| \leq(V(0)+B) / \gamma$ and

$$
\left|\int_{0}^{x(t)} f(\xi) d \xi\right|+\left|\int_{0}^{x(t)}\right| g(\xi)|d \xi| \leq B^{*} \text { for some } B^{*}>0
$$

By condition (3), it follows that $|x(t)|$ is bounded on $R^{+}$. This proves that (3) is sufficient.

Now we show that (3) is necessary. Suppose that condition (3) fails. To be definite, we assume that

$$
\int_{0}^{+\infty}[f(s)+|g(s)|] d s<+\infty .
$$

Let $\phi \in C([-h, 0], R)$ and $\phi(\xi)>k, \xi \in[-h, 0], x_{0}=\phi(0)$. Define

$$
\tilde{g}=\max \{g(\phi(\xi)): \xi \in[-h, 0]\}
$$


and

$$
y_{0}=2+h \tilde{g}+\int_{x_{0}}^{+\infty}[f(\xi)+g(\xi)] d \xi .
$$

Let $(x(t), y(t))$ be the solution of (5) with $x(\xi)=\phi(\xi), \xi \in[-h, 0]$ and $y(0)=y_{0}$. We claim that $y(s)>1$ for all $s \in R^{+}$.

Now suppose that there exists $t_{1}>0$ such that $y\left(t_{1}\right)=1$ and $y(s)>1$ on $\left[0, t_{1}\right)$. Consequently, $x(t)$ is increasing on $\left[0, t_{1}\right)$.

Case 1. Suppose that $t_{1} \leq h$. Integrate the second equation in (5) from 0 to $t_{1}$ to obtain

$$
\begin{aligned}
y\left(t_{1}\right) & =y_{0}-\int_{0}^{t_{1}} g(x(s-h)) d s-\int_{0}^{t_{1}} f(x(s)) x^{\prime}(s) d s \\
& \geq y_{0}-h \tilde{g}-\int_{x(0)}^{x\left(t_{1}\right)} f(\xi) d \xi \geq y_{0}-h \tilde{g}-\int_{x_{0}}^{+\infty} f(\xi) d \xi>1,
\end{aligned}
$$

a contradiction.

Case 2. Suppose that $t_{1}>h$. Integrating the second equation in (5) from 0 to $t_{1}$, we have

$$
\begin{aligned}
y\left(t_{1}\right) & =y_{0}-\int_{0}^{h} g(x(s-h)) d s-\int_{h}^{t_{1}}\left(g(x(s-h)) d s-\int_{0}^{t_{1}} f(x(s)) x^{\prime}(s) d s\right. \\
& \geq y_{0}-h \tilde{g}-\int_{0}^{t_{1}-h} g(x(s)) d s-\int_{0}^{t_{1}} f(x(s)) x^{\prime}(s) d s \\
& \geq y_{0}-h \tilde{g}-\int_{0}^{t_{1}-h} g(x(s)) x^{\prime}(s) d s-\int_{0}^{t_{1}} f(x(s)) x^{\prime}(s) d s \\
& \geq y_{0}-h \tilde{g}-\int_{x_{0}}^{+\infty} g(\xi) d \xi-\int_{x_{0}}^{+\infty} f(\xi) d \xi>1,
\end{aligned}
$$

a contradiction. Thus $y(t)>1$ on $R^{+}$and $x(t) \geq t+x_{0} \rightarrow+\infty$ as $t \rightarrow+\infty$. This completes the proof of Theorem 1 .

Theorem 2. If (2) is satisfied with $k=0$, then the zero solution of (5) is globally asymptotically stable if and only if (3) holds.

Proof. Suppose (2) and (3) hold with $k=0$. It is clear from the proof of Theorem 1 that the zero solution of $(5)$ is stable. Let $(x(t), y(t))$ be any solution of $(5)$ and $V_{1}(t)$ be defined in (6), then by (7) we have

$$
V_{1}^{\prime}(t) \leq-(N-1) F(x(t)) g(x(t)) / 2 N .
$$

This implies that $\lim _{t \rightarrow+\infty} \inf |x(t)|=0$.

Now suppose that $\lim _{t \rightarrow+\infty} \sup |x(t)|=\lambda>0$. Then there exist sequences $\left\{t_{n}\right\},\left\{t_{n}^{\prime}\right\}$ such that $0<t_{n}<t_{n}^{\prime}, t_{n} \rightarrow+\infty$ as $n \rightarrow+\infty$ and

$$
\left|x\left(t_{n}\right)\right|=\frac{\lambda}{3}, \quad\left|x\left(t_{n}^{\prime}\right)\right|=\frac{2 \lambda}{3}, \quad\left(\frac{\lambda}{3}\right)<|x(t)|<\frac{2 \lambda}{3} \quad \text { on }\left(t_{n}, t_{n}^{\prime}\right) .
$$

Let

$$
\widetilde{F}=\min \left\{F(\xi) g(\xi)\left|\frac{\lambda}{3} \leq\right| \xi \mid \leq \frac{2 \lambda}{3}\right\}
$$


Thus for $t \geq t_{n}^{\prime}$, we have

$$
\begin{aligned}
V_{1}(t) & \leq V_{1}\left(t_{1}\right)-(N-1) \int_{t_{1}}^{t} F(x(s)) g(x(s)) d s / 2 N \\
& \leq V_{1}\left(t_{1}\right)-(N-1) \sum_{j=1}^{n} \int_{t_{j}}^{t_{j}^{\prime}} F(x(s)) g(x(s)) d s / 2 N \\
& \leq V_{1}\left(t_{1}\right)-(N-1) \widetilde{F} \sum_{j=1}^{n}\left(t_{j}^{\prime}-t_{j}\right) / 2 N .
\end{aligned}
$$

Since (2) and (3) hold, there exists a constant $B>0$ such that

$$
|x(t)|+|y(t)| \leq B \text { for } t \geq t_{0} \text {. }
$$

Hence,

$$
\frac{\lambda}{3}=\frac{2 \lambda}{3}-\frac{\lambda}{3} \leq \int_{t_{j}}^{t_{j}^{\prime}}\left|x^{\prime}(s)\right| d s \leq B\left(t_{j}^{\prime}-t_{j}\right)
$$

and

$$
\lambda \leq 3 B\left(t_{j}^{\prime}-t_{j}\right) \text { for } j=1,2, \ldots
$$

This yields

$$
V_{1}(t) \leq V_{1}\left(t_{1}\right)-\frac{(N-1) \widetilde{F} n \lambda}{6 N B} \rightarrow-\infty \text { as } n \rightarrow+\infty,
$$

a contradiction. Therefore, $\lim _{t \rightarrow+\infty}|x(t)|=0$.

By (6) and (7), it follows that $V_{1}(t) \rightarrow C \geq 0$ as $t \rightarrow+\infty$ for somie constant $C$. Since $x(t) \rightarrow 0$ as $t \rightarrow+\infty$, we have $y^{2}(t) \rightarrow C$ as $t \rightarrow+\infty$. Now suppose $C>0$. Then there exists a constant $T>0$ such that $t \geq T$ implies $y^{2}(t) \geq$ $C / 2$. Without loss of generality, we may assume that $y(t) \geq(C / 2)^{1 / 2}$ for $t \geq$ $T$. Thus $x^{\prime}(t) \geq(C / 2)^{1 / 2}$ for $t \geq T$ so that $x(t) \geq x(T)+(C / 2)^{1 / 2}(t-T) \rightarrow$ $+\infty$ as $t \rightarrow+\infty$, a contradiction. This yields $\lim _{t \rightarrow+\infty} y(t)=0$. The proof of Theorem 1 shows that condition (3) is necessary for the global asymptotic stability of zero solution of (5). This completes the proof of Theorem 2 .

\section{ACKNOWLEDGMENT}

The author is very grateful to Professor T. A. Burton for his suggestion and encouragement to form this paper.

\section{REFERENCES}

1. T. A. Burton, On the Equation $x^{\prime \prime}(t)+f(x) h\left(x^{\prime}\right) x^{\prime}+g(x)=e(t)$, Ann. Mat. Pure Appl. 85 (1970), 277-286.

2. Stability and periodic solutions of ordinary and functional differential equations, Academic Press, Orlando, FL, 1985.

3. __ The generalized Liénard equation, SIAM J. Control Optim. 3 (1965), 223-230.

4. J. R. Graef, On the generalized Lienard equation with negative damping, J. Differential Equations 12 (1972), 34-62.

5. J. K. Hale, Sufficient conditions for stability and instability of autonomous functional differential equations, J. Differential Equations 1 (1965), 452-482.

6. T. Hara and T. Yoneyama, On the global center of generalized Liénard equation and its application to stability problems, Funkcial. Ekvac. 28 (1985), 171-192. 
7. Q. C. Huang and X. F. Shi, Global asymptotic behavior of solutions of second order differential equations, Acta Math. Sinica 27 (1984), 449-457.

8. J. F. Jiang, The asymptotic behavior of a class of second order differential equations with application to electrical circuit equations, J. Math. Anal. Appl. 149 (1990), 26-37.

9. N. N. Krasovskii, Stability of motion, Stanford Univ. Press, Stanford, CA, 1963.

10. N. Minorsky, Nonlinear oscillation, Van Nostrand, New York, 1962.

11. A. Somolinos, Periodic solutions of the sunflower equation, Quart. Appl. Math. 35 (1978), $465-478$.

12. J. Sugie, On the boundedness of solutions of the generalized Liénard equation without the signum condition, Nonlinear Anal. 11 (1987), 1391-1397.

13. _ On the generalized Liénard equation without the signum condition, J. Math. Anal. Appl. 128 (1987), 80-91.

14. G. Villari, On the qualitative behaviour of solutions of Liénard equation, J. Differential Equations 67 (1987), 269-277.

15. G. Villari and F. Zanolin, On a dynamical system in the Liénard plane. Necessary and sufficient conditions for the intersection with the vertical isocline and application, Funkcial. Ekvac. 33 (1990), 19-38.

16. T. Yoshizawa, Asymptotic behavior of solutions of differential equations, Differential Equations: Qualitative Theory (Szeged, 1984), Colloq. Math. Soc. János Bolyai, vol. 47, NorthHolland, Amsterdam, pp. 1141-1172.

Department of Mathematics, Southern Illinois University, Carbondale, Illinois 62901-4408 